\title{
Correction to: FastMM: an efficient toolbox for personalized constraint-based metabolic modeling
}

Gong-Hua Li ${ }^{1}$, Shaoxing Dai ${ }^{1}$, Feifei Han ${ }^{2}$, Wenxing Lii , Jingfei Huang ${ }^{1,3^{*}}$ and Wenzhong Xiao $24^{*}$

The original article can be found online at https://doi.org/10.1186/ s12859-020-3410-4.

* Correspondence: huangjf@mail.kiz. ac.cn; wenzhong.xiao@mgh.harvard. edu

${ }^{1}$ State Key Laboratory of Genetic Resources and Evolution, Kunming Institute of Zoology, Chinese Academy of Sciences, Kunming 650223, Yunnan, China

${ }^{2}$ Immue and Metabolic Computational Center,

Massachusetts General Hospital, Harvard Medical School, Boston, MA 02114, USA

Full list of author information is available at the end of the article

\section{Correction to: BMC Bioinformatics 21, 67 (2020)}

https://doi.org/10.1186/s12859-020-3410-4

Following publication of the original article [1], the authors identified an error in the author name of Wenxing Li.

1. Incorrect name:

Wenxin Li

2. Correct name:

Wenxing Li

The author group has been updated above and the original article [1] has been corrected.

\section{Author details}

'State Key Laboratory of Genetic Resources and Evolution, Kunming Institute of Zoology, Chinese Academy of Sciences, Kunming 650223, Yunnan, China. Immue and Metabolic Computational Center, Massachusetts General Hospital, Harvard Medical School, Boston, MA 02114, USA. ${ }^{3}$ Collaborative Innovation Center for Natural Products and Biological Drugs of Yunnan, Kunming 650223, Yunnan, China. ${ }^{4}$ Stanford Genome Technology Center, Stanford University, Palo Alto, CA 94304, USA.

Published online: 16 September 2020

\section{Reference}

1. Li G, et al. FastMM: an efficient toolbox for personalized constraint-based metabolic modeling. BMC Bioinformatics. 2020;21:67. https://doi.org/10.1186/s12859-020-3410-4. 